\title{
Plasmons in solar energy conversion
}

\author{
Anthony J. Morfa, Thomas H. Reilly III, Justin C. Johnson, and \\ Jao van de Lagemaat
}

Harnessing the oscillation of electrons on conductor surfaces can effectively enhance the performance of organic photovoltaic cells.

The need for affordable and large-scale, carbon-neutral energy sources has fueled a search for revolutionary concepts in solarto-electricity energy conversion. Studies often focus on doubling or tripling the conversion efficiency using third-generation concepts that circumvent traditional ${ }^{1}$ and inch closer to thermodynamic limits. ${ }^{2}$ Alternatively, researchers focus on making solar cells not quite as efficient but as cheap as paint. ${ }^{3}$ Such low-cost options are usually obtained by employing organic semiconductors or dye-sensitized systems. ${ }^{4}$

A common thread in all these systems is the tradeoff between efficient light absorption and charge collection. Cells engineered to absorb as much light as possible exhibit decreased efficiency, because increased path lengths strongly increase energy losses by recombination of oppositely charged electrical carriers. Photovoltaics engineered to exhibit less recombination absorb little light. A sweet spot can be found that has limited efficiency. To surpass this practical limit, new materials and approaches are needed.

A new way to increase solar-energy conversion is through surface plasmons, i.e., collective surface oscillations of conducting electrons in metal nanostructures that tend to trap optical waves near their surface. They enhance optical processes in their vicinity and are widely used in Raman spectroscopy, where enhancement factors can be many orders of magnitude. ${ }^{5}$ Analogous to Raman scattering, they enhance optical absorption, allowing for development of solar cells that circumvent the tradeoff between optical thickness and carrier transport. Yet, because strong recombination can occur at metal surfaces in contact with the active layer of a solar cell, attempts at this approach have not been very successful, except for some cases where they were used deliberately as recombination sites. ${ }^{6}$ We have recently succeeded in circumventing these issues by employing buffer layers between the plasmonically active

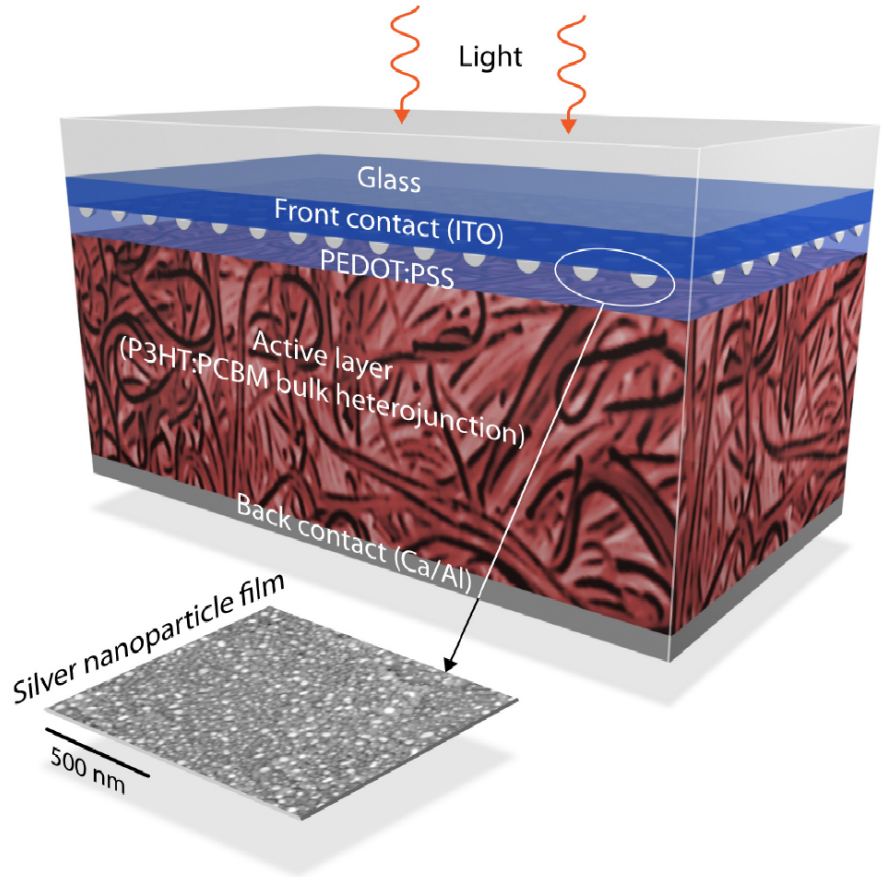

Figure 1. Plasmonically enhanced bulk-heterojunction solar cell. Silver nanoparticles are separated from the active layer by a poly(3,4ethylenedioxythiophene) poly(styrenesulfonate) (PEDOT:PSS) layer. ITO: Indium tin oxide. P3HT:PCBM: Poly-3-hexylthiophene:[6,6]phenyl-C61-butyric acid methyl ester. Ca/Al: Calcium/aluminum.

material and the active layer of the solar cell. ${ }^{7,8}$ We also employed a hybrid approach, where plasmonic effects potentially enable third-generation solar-energy conversion. ${ }^{9}$

Figure 1 shows one of the possible approaches to using plasmonic enhancement in organic solar cells. We vapor deposit silver particles on a transparent conducting front contact of indium tin oxide (ITO). We subsequently spin a layer of transparent conducting polymer, poly(3,4-ethylenedioxythiophene) poly(styrenesulfonate) (PEDOT:PSS), on top of this, followed by the active layer. Upon inclusion of silver particles, these cells 


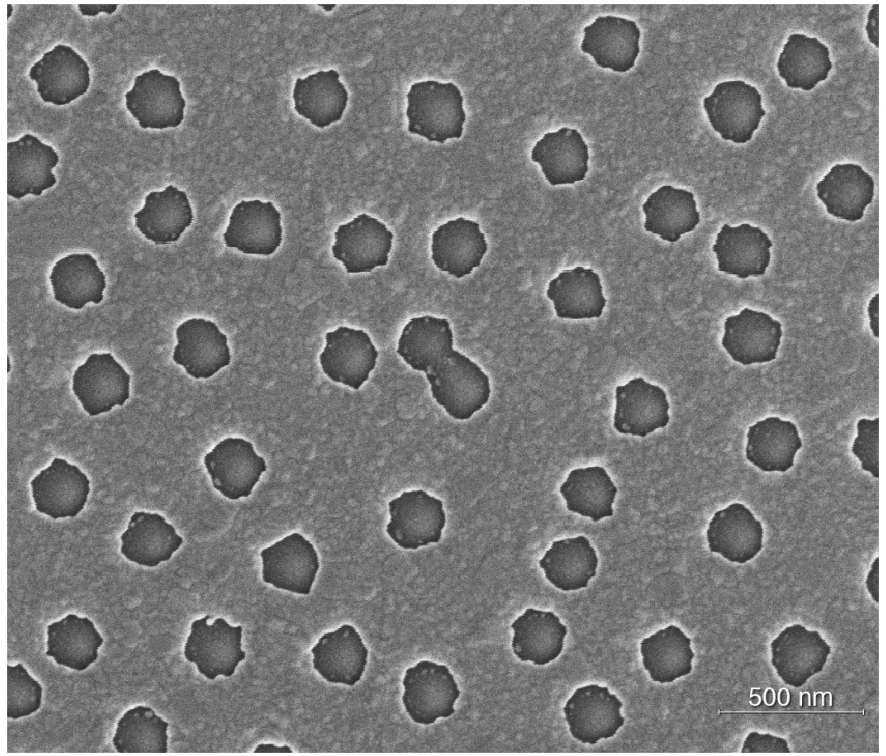

Figure 2. Scanning-electron micrograph of a random nanohole array in silver.

showed almost a doubling of their conversion efficiency. ${ }^{7}$ This increase in efficiency is due to higher absorption in the active layer of the device. The magnitude of the increase can be tuned by changing the thickness of the PEDOT:PSS layer. Our results were recently confirmed by another group using a different silver-deposition method, ${ }_{10}^{10}$ and we have repeated these experiments on random nanohole arrays in silver, omitting ITO. ${ }^{8}$ We (and others) also attempted to include the silver particles inside the active layer as that would result in an even better geometry for optical enhancement. However, such an approach is presently hampered by excessive recombination or only very limited enhancements. ${ }^{11}$ We are working on remedying this.

Next to direct absorbance enhancement, we have studied the ultrafast photophysics of molecular semiconductors on surface-plasmon-active electrodes using transient absorbance measurements. ${ }^{9}$ In particular, we studied a system consisting of pentacene that was vapor deposited on random silver nanohole $(\mathrm{AgNH})$ arrays that were fabricated using colloidal lithography. ${ }^{8}$ Pentacene is an interesting molecule because it is a candidate for 'singlet fission' (SF), in which a single (singlet) exciton is converted into two (triplet) excitons. ${ }^{12}$ Just like multiexciton generation in quantum dots, ${ }^{13}$ this can be used to increase the solar-cell conversion efficiency over the Shockley-Queisser limit. ${ }^{1}$ Pentacene's energy levels are close to being suitable for this process, with the triplet having approximately half the energy of the singlet. Coupling exciton states on the pentacene with surface plasmons might alter the excited-state dynamics

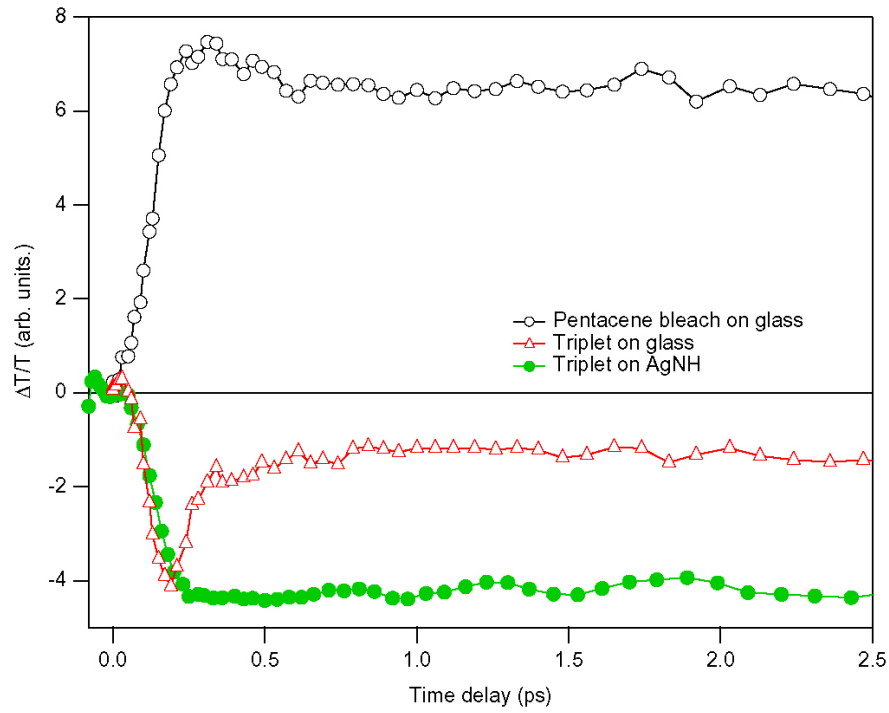

Figure 3. Ultrafast triplet-decay kinetics for pentacene on silvernanohole $(\mathrm{AgNH})$ arrays. $\triangle \mathrm{T} / \mathrm{T}$ : Relative change in transmittance.

as well as the relative rates of exciton fission and fusion to produce a larger, long-lived multiexciton yield. Figure 3 shows the ultrafast triplet-decay kinetics of pentacene in the presence and absence of an AgNH array. Clearly, the array alters the triplet yield and decay kinetics significantly. From this data, we cannot conclusively say that SF occurs, but it provides a tantalizing suggestion.

We have demonstrated plasmonic enhancement of solarenergy conversion in organic solar cells. Inclusion of a plasmonically active front electrode using a layer of silver nanoparticles or a random nanohole array increases the cell's current generation by absorbance enhancement through plasmonic near-field effects. Such enhanced photoconversion shows great promise for organic and other solar-cell technologies. We are currently attempting to include the plasmon-active materials inside the active layer, as well as studying the enhancement mechanism more closely. We have also shown that the proximity of molecular excitons to surface plasmons can greatly enhance excitedstate dynamics. These results are potentially very important for third-generation photogeneration systems.

We thank Al Hicks for help with Figure 1 and Bobby To for the scanning-electron microscopy image. J.C.J. acknowledges support from the Hydrogen Fuel Initiative of the US Department of Energy (Office of Science, Basic Energy Sciences). J.L. acknowledges the Photochemistry and Radiation Research Program of the US Department of Energy 
(Office of Science, Basic Energy Sciences, Division of Chemical Sciences, Geosciences and Biosciences) for support. This work was supported by the US Department of Energy under contract DE-AC3608GO28308 with the National Renewable Energy Laboratory.

\section{Author Information}

\section{Anthony J. Morfa}

Nanoscience Laboratory

University of Melbourne

Melbourne, Australia

Anthony Morfa was a graduate student at the University of Colorado at Boulder under the tutelage of Jao van de Lagemaat. He demonstrated plasmon enhancement in organic bulk-heterojunction solar cells. He is currently a postdoctoral fellow.

\section{Thomas H. Reilly III, Justin C. Johnson, and} Jao van de Lagemaat

Chemical Sciences and Biosciences Center

National Renewable Energy Laboratory

Golden, CO

http:/ / www.nrel.gov/basic_sciences/technology_staff.cfm/

tech $=14 / \mathrm{ID}=24$

http:/ / www.nrel.gov/basic_sciences/technology.cfm/

tech $=14$ /

Thomas Reilly is a postdoc studying how surface plasmons enhance next-generation photovoltaic materials. His research centers on using nanofabrication methods to explore the incorporation of plasmonic materials in polymer and small-molecule solar cells. His dissertation focused on generating nanofabricated substrates for surface-plasmon-enhanced molecular spectroscopy.

Justin Johnson is a research scientist specializing in ultrafast spectroscopy. His previous research has included investigations of single-nanowire lasers and waveguides using nearfield microscopy, nonlinear optical spectroscopy of semiconductor nanocrystals, and spectroscopic studies of exciton fission in novel organic chromophores.

Jao van de Lagemaat is a senior research scientist. He is currently researching tunneling-induced luminescence and plasmonresonance imaging of individual quantum dots and quantumdot arrays as well as the use of plasmonic-enhancement effects in solar-energy-conversion systems.

\section{References}

1. W. Shockley and H. Queisser, Detailed balance limit of efficiency of $p$ - $n$ junction solar cells, J. Appl. Phys. 32, p. 510, 1961. doi:10.1063/1.1736034

2. A. Nozik, Quantum dot solar cells, Physica E 14 (1-2), pp. 115-120, 2002 doi:10.1016/S1386-9477(02)00374-0

3. H. Hoppe and N. S. Sariciftci, Organic solar cells: an overview, J. Mater. Res. 19 (7), pp. 1924-1945, 2004. doi:10.1557/JMR.2004.0252

4. A. Frank, N. Kopidakis, and J. van de Lagemaat, Electrons in nanostructured $\mathrm{TiO}_{2}$ solar cells: transport, recombination and photovoltaic properties, Coordin. Chem. Rev 248, pp. 1165-1179, 2004. doi:10.1016/j.ccr.2004.03.015

5. J. Jiang, K. Bosnick, M. Maillard, and L. Brus, Single molecule Raman spectroscopy at the junctions of large Ag nanocrystals, J. Phys. Chem. B 107 (37), pp. 9964-9972, 2003. doi:10.1021/jp034632u

6. B. Rand, P. Peumans, and S. Forrest, Long-range absorption enhancement in organic tandem thin-film solar cells containing silver nanoclusters, J. Appl. Phys. 96 (12), pp. 7519-7526, 2004. doi:10.1063/1.1812589

7. A. Morfa, K. Rowlen, T. Reilly, M. Romero, and J. van de Lagemaat, Plasmonenhanced solar energy conversion in organic bulk heterojunction photovoltaics, Appl. Phys. Lett. 92 (1), p. 013504, 2008. doi:10.1063/1.2823578

8. T. Reilly, J. van de Lagemaat, R. Tenent, A. Morfa, and K. Rowlen, Surfaceplasmon enhanced transparent electrodes in organic photovoltaics, Appl. Phys. Lett. 92 (24), p. 243304, 2008. doi:10.1063/1.2938089

9. J. Johnson, T. Reilly, A. Kanarr, and J. van de Lagemaat, The ultrafast photophysics of pentacene coupled to surface plasmon active nanohole films, J. Phys. Chem. C 113 (16), pp. 6871-6877, 2009. doi:10.1021/jp901419s

10. S.-S. Kim, S.-I. Na, J. Jo, D.-Y. Kim, and Y.-C. Nah, Plasmon enhanced performance of organic solar cells using electrodeposited Ag nanoparticles, Appl. Phys. Lett. 93 (7) p. 073307, 2008. doi:10.1063/1.2967471

11. K. Kim and D. Carroll, Roles of Au and Ag nanoparticles in efficiency enhancement of poly(3-octylthiophene)/C-60 bulk heterojunction photovoltaic devices, Appl. Phys. Lett. 87 (20), p. 203113, 2005. doi:10.1063/1.2128062

12. I. Paci, J. Johnson, X. Chen, G. Rana, D. Popović, D. David, A. Nozik, M. Ratner, and J. Michl, Singlet fission for dye-sensitized solar cells: can a suitable sensitizer be found?, J. Am. Chem. Soc. 128 (51), pp. 16546-16553, 2006. doi:10.1021/ja063980h

13. R. Ellingson, M. Beard, J. Johnson, P. Yu, O. Micic, A. Nozik, A. Shabaev, and A. Efros, Highly efficient multiple exciton generation in colloidal PbSe and PbS quantum dots, Nano Lett. 5, pp. 865-871, 2005. doi:10.1021/nl0502672 